\title{
Nucleic Acid-Based Therapeutics Relevant to Neuroimmune Conditions
}

\author{
Ariele L. Greenfield ${ }^{1}$ • Stephen L. Hauser ${ }^{1}$ (D) \\ Published online: 13 May 2019 \\ (C) The American Society for Experimental NeuroTherapeutics, Inc. 2019
}

Treatments for autoimmune neurologic disease have become increasingly powerful, yet have not been able to eliminate disease activity altogether. Underlying neurodegeneration may start early on in the course of neuroimmune diseases such as multiple sclerosis (MS), making early aggressive treatment of immunologic reservoirs an increasingly recognized treatment strategy. There is a need for more specific therapeutic targets as well. Novel approaches to treating these conditions, from cell-based therapies to gene therapy, show promise for filling these unmet needs (Table 1). Nucleic acid-based therapies have the potential to more precisely target specific populations of target cells and more precisely correct specific gene regulatory abnormalities, avoiding wider-scale immunosuppression and off-target effects.

\section{CAR-T and CAAR-T Cells}

Chimeric antigen receptor $\mathrm{T}(\mathrm{CAR}-\mathrm{T})$ cells link a $\mathrm{T}$ cell activating domain to an immunoglobulin heavy and light chain pair that defines the $\mathrm{T}$ cell receptor (TCR) specificity for a particular protein [1]. These receptors can be made using CRISPR-based genome editing [2] or viral vectors, such as lentivirus, adenovirus, and adeno-associated virus [3].

One of the most exciting developments in nucleic acidbased therapies for autoimmune disease is the invention of chimeric autoantibody receptor T (CAAR-T) cells. CAAR-T therapy has shown preliminary promise in pemphigus vulgaris. These genetically engineered $\mathrm{T}$ cells express a TCR

Electronic supplementary material The online version of this article (https://doi.org/10.1007/s13311-019-00740-5) contains supplementary material, which is available to authorized users.

Stephen L. Hauser

stephen.hauser@ucsf.edu

1 UCSF Weill Institute for Neurosciences, Department of Neurology, University of California, San Francisco, San Francisco, CA, USA with an extracellular domain displaying not an immunoglobulin, but an autoantigen - in this case desmoglein [4]. Without inducing broad immunosuppression or destruction of an entire class of immune cells, this therapy causes $T$ cells to specifically lyse B cells that bind the desmoglein protein. This strategy has not yet been used in neuroinflammatory disease; however, we speculate that this could be beneficial for diseases thought to involve single antigen specificity: paraneoplastic and autoimmune encephalitides with single epitopes such as NR1 in anti-NMDA-receptor encephalitis, and AQP4 in neuromyelitis optica. Targeting specific B cells in this way could provide a treatment strategy that leaves the immune system largely intact. Additionally, CAAR-T has the ability to traverse the blood-brain barrier more freely [5] than monoclonal antibodies - which only penetrate $0.1 \%$ [6] - potentially depleting pathogenic immune cells within the central nervous system (CNS) more thoroughly. Targeting antigen-specific $\mathrm{T}$ cell populations may be beneficial as well. $\beta$-synuclein-reactive $\mathrm{T}$ cells, for example, have been reported in progressive MS [7] and could be one potential antigen-specific $T$ cell subpopulation to selectively deplete.

There is even the potential to program CAR-T cells to only activate when presented with multiple signals - allowing for even greater tissue-specific and context-specific action. Activation of one surface chimeric receptor can activate transcription and expression of a second chimeric receptor which is in turn linked to the TCR activation signal. In this way, these $\mathrm{T}$ cells only kill a target cell meeting dual criteria for both chimeric receptors [8]. Bispecific CAR-T cellsprogrammed to respond to either one or another signalhave demonstrated proof of concept in preclinical studies [9] and are currently in clinical trials for conditions such as dualantigen (CD19 and BCMA) multiple myeloma. When targeting CAR-T cells to the CNS for glioma, engineering "next-generation" CAR-T cells to require multiple signals for their activation and to secrete cytokines to support their proliferation and survival in the CNS are attractive goals [10]. This strategy has the potential, for example, to enable anti- 


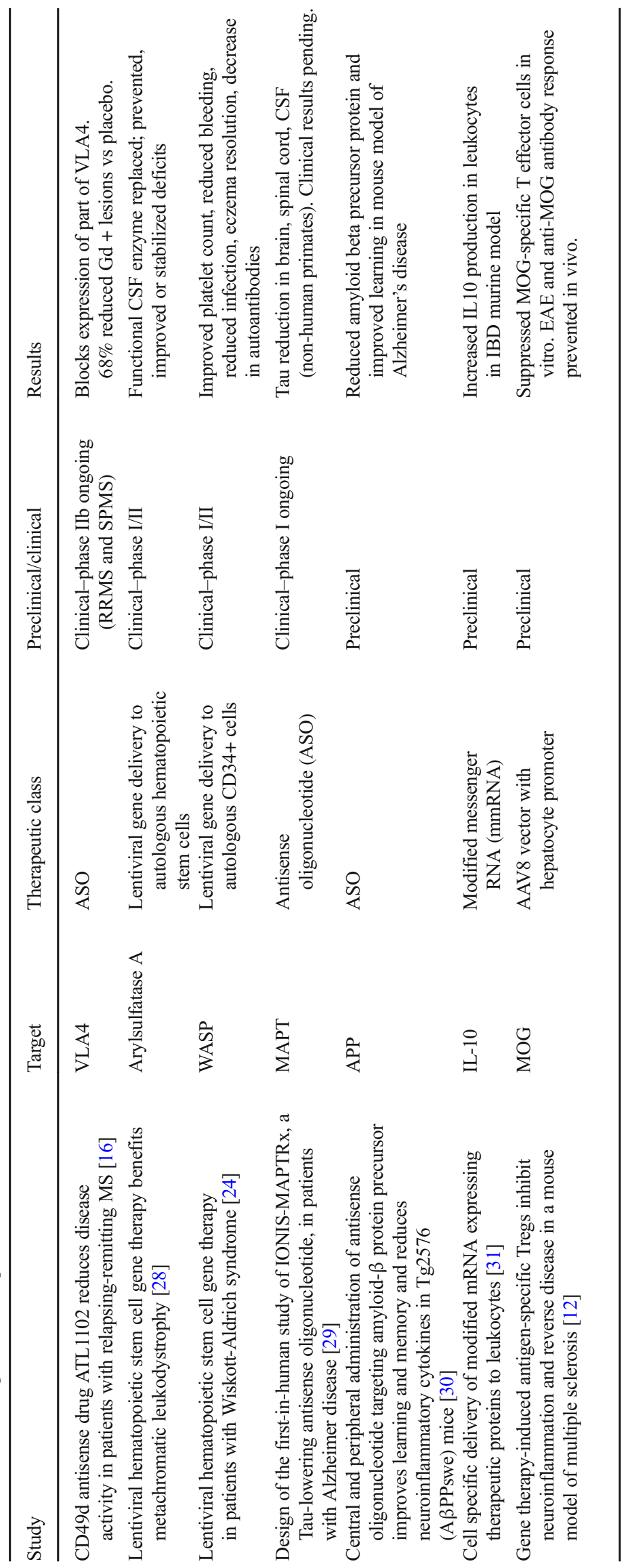


idiotype CAR-T cells to remove only B cells that display both a pro-inflammatory signal and an autoantigen-specific immunoglobulin. Recently, CRISPR-edited B cell receptors have been generated, enabling directing $\mathrm{B}$ cells towards a specific antigen as well [11].

\section{AAV-Treated Hepatocytes to Induce Tregs}

Adenoviral therapy has been used in animal models to indirectly stimulate tolerogenic regulatory $\mathrm{T}$ cells (Tregs). Experimental autoimmune encephalomyelitis (EAE) is a mouse model of MS in which an immune response is directed against myelin oligodendrocyte glycoprotein (MOG), a glycoprotein on the outer lamellae of myelin. When mice were treated with a hepatocyte-targeting AAV8 encoding fulllength MOG, expression of full-length MOG was induced in hepatocytes. Tregs specific for the MOG protein were in turn generated by the presence of MOG on otherwise normal hepatocytes. Then, when attempting to induce EAE, the effector $T$ cell population that otherwise would have been produced by the EAE model was suppressed. The EAE syndrome did not develop in these mice. When late-stage EAE had already set in before treating with AAV8-MOG, the course was even shown to improve [12]. Indirect induction of antigen-specific Tregs in this setting was shown to be three times more effective than transfer of naïve polyclonal Tregs [12]. Although this is strategy provides important evidence regarding the role of Tregs, translating this approach directly is unlikely given the potential for off-target effects when expressing a CNS protein in non-CNS tissue. More likely, a form of this approach could be used as an ex vivo method to tolerize autologous T cells. One challenge with antigen-specific cell-depleting therapies in neuroimmune conditions is that for many conditions, the antigenic targets of B and T cells in the CNS are unknown. Further, even in conditions for which an antigenic target has been identified, it is not always clear that the antigen-directed response is primarily responsible for the disease.

\section{Ex Vivo Expanded Tregs and CAR-Tregs}

Engineering the DNA of the TCR within T cells of a regulatory phenotype, the goal is to deliver greater immune tolerance to a given tissue. Tissue homing specificity is provided by the chimeric TCR, and the self-tolerant tone is provided by the cell's Treg phenotype (i.e., FOXP3 expression, secretion of antiinflammatory cytokines including interleukin-10). This strategy is currently being tested in autoimmune disease outside the nervous system. The rationale is that Tregs are deficient in inflamed target tissues of autoimmune disease, such as the pancreas in type 1 diabetes. Tregs in T1DM can be "unstable" and paradoxically secrete pro-inflammatory effector $\mathrm{T}$ cell molecules such as interferon gamma. Clonal Tregs specific for an antigen on the target tissue are far more effective in animal models than polyclonal Tregs [13]. In this method, the antigen specificity or exact targets of autoimmune $B$ and $T$ cells does not need to be discovered. Rather, Treg homing to the tissue target aims to re-train the microenvironment to decrease the inflammatory cascade. This strategy has undergone a phase 1 trial in type 1 diabetes [14] and will be completing a phase 2 this year. MOG-specific CAR-Tregs have been shown to suppress EAE [15]. Tregs can also induce long-lasting immune tolerance by recruiting other cell populations. Even if single target antigens are not identified in all neuroinflammatory conditions, allowing Tregs to home to the target tissue by engineering specificity of the TCR for a protein in that tissue may induce beneficial anti-inflammatory tone. An advantage to the CAR-Treg approach in CNS neuroimmune conditions is that the pathogenic antigen-directed response does not need to be elucidated first. This could also be important if, for example, in certain CNS autoimmune conditions, such as MS or lupus, it is found that there are private, e.g., patient-specific, or multiple antigen targets of the autoimmune response.

\section{Antisense Oligonucleotides}

An antisense oligonucleotide (ASO) against CD49d, ATL1102, has been tested in clinical trials of MS patients [16]. To our knowledge, this is the first use of an ASO in neuroimmune disease. CD49d encodes part of an integrin dimer targeted by a highly effective existing MS therapy. The existing monoclonal antibody therapy, natalizumab, blocks the VLA4 integrin found on all leukocytes except neutrophils from binding to vascular and mucosal adhesion molecules [17]. In this way, migration into the CNS is reduced [18, 19]. In this preliminary phase 2,12 -week study, the CD $49 \mathrm{~d}$ ASO was shown to reduce the number of active MS brain lesions that convert to "black hole" areas of tissue loss. The volume of new inflammatory MS lesions was also reduced by $84 \%$, compared to natalizumab's previously reported $50 \%$ reduction at 12 weeks [16]. The authors of this report speculate that a potential, albeit unproven, advantage of this therapy might be augmented safety above that of the broad antiVLA-4 antibody reactivity of natalizumab, in terms of a lower risk for progressive multifocal leukoencephalopathy (PML). One proposed mechanism for why an ASO reducing VLA4 may have a lower risk of PML is that the natalizumab antibody induces release of $\mathrm{CD} 34+$ hematopoietic precursor cells and CD19+ immature B cells. These cells can carry low copy levels of JC virus even in JCV Ab negative patients, and can go on to proliferate, yielding augmented JCV levels. ATL1102 does not cause this increase in CD34+ cells. There may also be differences in inflammatory response and rebound MS activity risk with this approach. As it works by lowering the level 
of surface integrin $\sim 50 \%$, it does not stimulate intracellular signaling cascades in the way a monoclonal antibody can. This could be a new strategy for inhibiting targets within the CNS, in which antibodies penetrate at a very low rate. For example, blocking CNS targets on microglia, such as CD22 [20], may promote improved tissue homeostasis in the setting of neurologic injury.

\section{Lentiviral Gene Therapy}

Monogenic neuroimmune diseases have recently been addressed with gene therapy, for example, in patients with adrenoleukodystrophy. These patients harbor loss of function mutations in the ABCD1 gene. Although this condition has been treated previously with allogeneic stem cell transplantation, the lack of an HLA-matched bone marrow donor can be problematic. There is now evidence that ex vivo lentiviral transduction of ABCD1 DNA into autologous CD34+ cells followed by infusion into patients may be safe and beneficial [21, 22]. This therapy was considerably safer than allogeneic stem cell transplantation-with zero versus 18\% 1-year mortality [22]. Wiskott-Aldrich syndrome, which can manifest with multiple autoimmune syndromes in the setting of T cell immunodeficiency, including CNS vasculitis [23] has been studied with lentiviral gene replacement [24] and shown to induce stable engraftment of cells with the replaced gene as well. Gene therapy has the potential to expand to other conditions involving a few or single key genes. However, this strategy will likely prove challenging in diseases that are highly polygenic, involving small effects from multiple genetic risk factors in combination with environmental contributions [25].

\section{Safety and Efficacy Considerations}

CAR-T cell therapy has the potential to cross the blood brain barrier and target cells within the CNS, as CAR-T DNA after peripheral infusion has readily been found in the CSF [26]. CAR-T cell-related encephalopathy syndrome and cytokine release syndrome [13] are serious adverse effects related to treating tumors with CAR-T. Off-target effects and the degree of proliferation of infused cell populations remain considerations as well: for cell populations that could mature to become long-lived, including a "deplete" molecular switch that can be activated at a later time may be an important element of therapy for autoimmune indications. Certain vectors used in gene therapy, such as adenovirus, invoke an immune response from the patient [21] which also can limit efficacy. Off-target effects of lentiviral therapies, such as inducing oncogenes and promoting malignancy, are concerns but have been less of a concern with newer lentiviruses used for delivery. There are particular risks of ASOs as well. For example, although the
CD49d ASO described is cleared rapidly from the blood into lymphoid tissues (bone, spleen, lymph nodes) by lymphocytes expressing VLA4, ASOs have been associated with deposition in bone as well [27]. $20 \%$ of patients in [16] also experienced an increase in liver enzymes.

\section{Summary}

New nucleic acid modifying therapies are emerging in the treatment of neuroimmune disease. These treatments have the potential for increased tissue specificity, greater tissue penetrance, and for correcting genetic defects in pro-inflammatory pathways that were not previously possible. As the field progresses, the shortand long-term safety profile of these therapies, and ways of monitoring for off-target effects of genetically engineered human cells and human transcripts will be important.

Required Author Forms Disclosure forms provided by the authors are available with the online version of this article.

\section{References}

1. June, C. H. \& Sadelain, M. Chimeric Antigen Receptor Therapy. New England Journal of Medicine 379, 64-73, doi:https://doi.org/ 10.1056/NEJMra1706169 (2018).

2. Ren, J. et al. A versatile system for rapid multiplex genome-edited CAR T cell generation. Oncotarget 8, 17002-17011, doi:https://doi. org/10.18632/oncotarget.15218 (2017).

3. Zhang, C., Liu, J., Zhong, J. F. \& Zhang, X. Engineering CAR-T cells. Biomarker research 5, 22-22, doi:https://doi.org/10.1186/ s40364-017-0102-y (2017).

4. Ellebrecht, C. T. et al. Reengineering chimeric antigen receptor T cells for targeted therapy of autoimmune disease. Science (New York, N.Y.) 353, 179-184, doi:https://doi.org/10.1126/science. aaf6756 (2016).

5. O'Rourke, D. M. et al. A single dose of peripherally infused EGFRvIII-directed CAR T cells mediates antigen loss and induces adaptive resistance in patients with recurrent glioblastoma. Science translational medicine 9, doi:https://doi.org/10.1126/scitranslmed. aaa0984 (2017)

6. Yu, Y. J. \& Watts, R. J. Developing therapeutic antibodies for neurodegenerative disease. Neurotherapeutics : the journal of the American Society for Experimental NeuroTherapeutics 10, 459472, doi:https://doi.org/10.1007/s13311-013-0187-4 (2013).

7. Lodygin, D. et al. beta-Synuclein-reactive T cells induce autoimmune CNS grey matter degeneration. Nature 566, 503-508, doi: https://doi.org/10.1038/s41586-019-0964-2 (2019).

8. Roybal, K. T. \& Lim, W. A. Synthetic Immunology: Hacking Immune Cells to Expand Their Therapeutic Capabilities. Annual review of immunology 35, 229-253, doi:https://doi.org/10.1146/ annurev-immunol-051116-052302 (2017).

9. Zah, E., Lin, M.-Y., Silva-Benedict, A., Jensen, M. C. \& Chen, Y. Y. T Cells Expressing CD19/CD20 Bispecific Chimeric Antigen Receptors Prevent Antigen Escape by Malignant B Cells. Cancer 
immunology research 4, 498-508, doi:https://doi.org/10.1158/ 2326-6066.CIR-15-0231 (2016).

10. Petersen, C. T. \& Krenciute, G. Next Generation CAR T Cells for the Immunotherapy of High-Grade Glioma. Frontiers in Oncology 9, doi:https://doi.org/10.3389/fonc.2019.00069 (2019).

11. Voss, J. E. et al. Reprogramming the antigen specificity of B cells using genome-editing technologies. eLife $\mathbf{8}$, e42995, doi:https://doi. org/10.7554/eLife.42995 (2019).

12. Keeler, G. D. et al. Gene Therapy-Induced Antigen-Specific Tregs Inhibit Neuro-inflammation and Reverse Disease in a Mouse Model of Multiple Sclerosis. Molecular therapy : the journal of the American Society of Gene Therapy 26, 173-183, doi:https://doi. org/10.1016/j.ymthe.2017.09.001 (2018).

13. Neelapu, S. S. et al. Chimeric antigen receptor T-cell therapy assessment and management of toxicities. Nature Reviews Clinical Oncology 15, 47, doi: https://doi.org/10.1038/nrclinonc.2017.148

14. Bluestone, J. A. et al. Type 1 diabetes immunotherapy using polyclonal regulatory T cells. Science translational medicine 7, 315ra189, doi:https://doi.org/10.1126/scitranslmed.aad4134 (2015).

15. Fransson, M. et al. CAR/FoxP3-engineered T regulatory cells target the CNS and suppress EAE upon intranasal delivery. Journal of neuroinflammation 9, 112-112, doi:https://doi.org/10.1186/17422094-9-112 (2012).

16. Limmroth, V., Barkhof, F., Desem, N., Diamond, M. P. \& Tachas, G. CD49d antisense drug ATL1102 reduces disease activity in patients with relapsing-remitting MS. Neurology 83, 1780-1788, doi: https://doi.org/10.1212/wnl.0000000000000926 (2014).

17. Tysabri (natalizumab) [package insert]. Biogen, Research Triangle Park, NC. (2004).

18. Parker Harp, C. R. et al. VLA4 expression by B cells enables B cellrestricted antigen presentation to support CD4 T cell driven central nervous system autoimmunity. The Journal of Immunology 200, 100.112 (2018).

19. Schneider-Hohendorf, T. et al. VLA-4 blockade promotes differential routes into human CNS involving PSGL-1 rolling of T cells and MCAM-adhesion of $\mathrm{T}<\mathrm{sub}>\mathrm{H}</$ sub $>17$ cells. The Journal of Experimental Medicine 211, 1833-1846, doi:https://doi.org/10. 1084/jem.20140540 (2014).

20. Pluvinage, J. V. et al. CD22 blockade restores homeostatic microglial phagocytosis in ageing brains. Nature 568, 187-192, doi:https://doi.org/10.1038/s41586-019-1088-4 (2019).
21. High, K. A. \& Brenner, M. K. in Harrison's Principles of Internal Medicine, 20e (eds J. Larry Jameson et al.) (McGraw-Hill Education, 2018).

22. Gene Therapy for Cerebral Adrenoleukodystrophy. New England Journal of Medicine 378, 490-491, doi:https://doi.org/10.1056/ NEJMc1715427 (2018).

23. Mahlaoui, N. et al. Characteristics and outcome of early-onset, severe forms of Wiskott-Aldrich syndrome. Blood 121, 1510 1516, doi:https://doi.org/10.1182/blood-2012-08-448118 (2013).

24. Aiuti, A. et al. Lentiviral hematopoietic stem cell gene therapy in patients with Wiskott-Aldrich syndrome. Science (New York, N.Y.) 341, 1233151-1233151, doi:https://doi.org/10.1126/science. 1233151 (2013).

25. Cotsapas, C. \& Mitrovic, M. Genome-wide association studies of multiple sclerosis. Clinical \& translational immunology 7, e1018e1018, doi:https://doi.org/10.1002/cti2.1018 (2018).

26. Rheingold, S. R. et al. Efficient Trafficking of Chimeric Antigen Receptor (CAR)-Modified T Cells to CSF and Induction of Durable CNS Remissions in Children with CNS/Combined Relapsed/ Refractory ALL. Blood 126, 3769-3769 (2015).

27. Geary, R. S., Norris, D., Yu, R. \& Bennett, C. F. Pharmacokinetics, biodistribution and cell uptake of antisense oligonucleotides. Advanced Drug Delivery Reviews 87, 46-51, doi:https://doi.org/ 10.1016/j.addr.2015.01.008 (2015).

28. Biffi, A. et al. Lentiviral hematopoietic stem cell gene therapy benefits metachromatic leukodystrophy. Science (New York, N.Y.) 341, 1233158, doi:https://doi.org/10.1126/science.1233158 (2013).

29. Mignon, L. et al. Design of the First-in-Human Study of IONISMAPTRx, a Tau-lowering Antisense Oligonucleotide, in Patients With Alzheimer Disease (S2.006). Neurology 90, S2.006 (2018).

30. Farr, S. A., Erickson, M. A., Niehoff, M. L., Banks, W. A. \& Morley, J. E. Central and peripheral administration of antisense oligonucleotide targeting amyloid- $\beta$ protein precursor improves learning and memory and reduces neuroinflammatory cytokines in $\mathrm{Tg} 2576$ (AßPPswe) mice. Journal of Alzheimer's disease : JAD 40, 1005-1016, doi:https://doi.org/10.3233/JAD-131883 (2014).

31. Veiga, N. et al. Cell specific delivery of modified mRNA expressing therapeutic proteins to leukocytes. Nature Communications $\mathbf{9}$, 4493, doi:https://doi.org/10.1038/s41467-018-06936-1 (2018).

Publisher's Note Springer Nature remains neutral with regard to jurisdictional claims in published maps and institutional affiliations. 